\title{
TWITTER L'ACTUALITÉ : USAGES ET RÉSEAUTAGE CHEZ LES JOURNALISTES FRANÇAIS
}

\begin{abstract}
Arnaud Mercier ${ }^{1}$
De façon à cerner au mieux les usages des réseaux sociaux par les journalistes français pour couvrir l'actualité, nous avons réalisé, en janvier 2012, une enquête par questionnaire dans une vingtaine de rédactions nationales et via notre site. Dans le même temps, nous avons construit un corpus de 600 comptes Twitter et 600 pages Facebook de journalistes publiant de façon à peu près régulière et qui possédaient le plus d'inscrits. En croisant ces données déclaratives et d'observation, nous cherchions à établir une forme de cartographie des divers usages. Les résultats obtenus nous conduisent ici à insister davantage sur Twitter, car ce réseau apparaît bien plus pratiqué par ces journalistes que Facebook.
\end{abstract}

Parmi les innovations produites par l'avènement d'Internet figurent en bonne place les médias sociaux, englobant des réseaux sociaux comme Facebook, LinkedIn ou TumblR ; des réseaux sociaux basés sur la géolocalisation comme Foursquare; des sites de micro-blogging dont le plus connu est Twitter ; ou des réseaux de partage de photos (Pinterest, Instagram) ou de vidéos (You Tube ou Dailymotion). Même si les usages journalistiques sont encore récents, quelques données sont déjà disponibles.

1 Arnaud Mercier et Professeur en Sciences de l'information et de la communication UFR Sciences humaines et arts à l'Université de Lorraine.

Recherches en communication, $\mathrm{n}^{\circ} 39$ (2013). 
Ainsi, en septembre 2011, une étude de l'agence de marketing digital, Arketi, a été conduite par sondage auprès de journalistes américains travaillant dans la presse spécialisée/professionnelle pour comprendre comment ils utilisaient les médias sociaux. Premier constat, le recours à l'Internet est omniprésent : $64 \%$ des journalistes interrogés déclarent y passer plus de 20 heures par semaine et $21 \%$ plus de 40 heures, pour s'informer soi-même (98\%) ou trouver des idées ou des sources pour ses propres articles $(91 \%)$, ou pour fréquenter les médias sociaux (69\%). Beaucoup ont plusieurs comptes sur diverses plateformes. Puisque les journalistes interrogés sont aussi dans la presse professionnelle, le premier réseau qu'ils utilisent est celui dédié au business, à savoir LinkedIn ( $92 \%$ ont un compte). Facebook et Twitter sont ensuite au coude à coude ( $85 \%$ et $84 \%)$. Pour Twitter, de tels chiffres sont très éloignés de ceux concernant la population en général. Beaucoup d'autres réseaux sont également représentés dans cet échantillon de journalistes, mais avec en dernier celui de géolocalisation Foursquare, dont l'usage journalistique est encore à trouver, puisque $14 \%$ seulement y ont un compte.

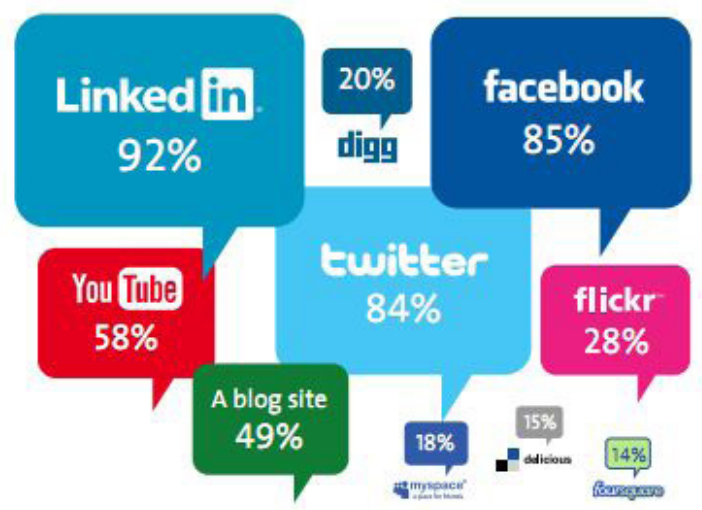

Figure 1. Usages des réseaux sociaux par les journalistes américains (en 2011)

Une présence aussi massive sur les médias sociaux ne signifie pas un bouleversement complet de la façon de préparer un article. 99\% des journalistes disent prendre leurs informations « à la source », dans l'entreprise, $80 \%$ par l'intermédiaire des relations publiques, et $77 \%$ par les communiqués de presse. En regard, 56\% trouvent leurs infos sur des blogs, $44 \%$ sur les sites de micro-blogging comme Twitter et 39\% via des réseaux sociaux comme Facebook ou LinkedIn. 
Si le poids des réseaux sociaux dans l'interaction entre journalistes, sources et lecteurs n'est pas encore majeur et décisif, néanmoins, la progression des usages informatifs de ces médias sociaux et la croissance rapide du nombre de titulaires d'un compte laissent deviner un support d'avenir pour l'information, surtout en ce qui concerne Twitter (fondé en août 2006), dont les caractéristiques particulièrement bien adaptées à certains aspects de l'information en ligne en font un outil professionnellement utile (Ahmad, 2010).

Ces réseaux sociaux commencent donc à être utilisés comme un lieu de repérage de sources potentielles pour tout à la fois faire une veille sur les informations diffusées par les concurrents, identifier des sujets qui font le buzz et alimenter en éventuels témoignages des articles. En 2011, la Digital Journalism Study a ainsi, pour la première fois, jugé opportun d'interroger les journalistes enquêtés sur leurs usages des réseaux sociaux comme sources. $47 \%$ utilisent Twitter et $35 \%$ Facebook.

\section{Figure 4. Please select which of the following you use when SOURCING new story leads?}

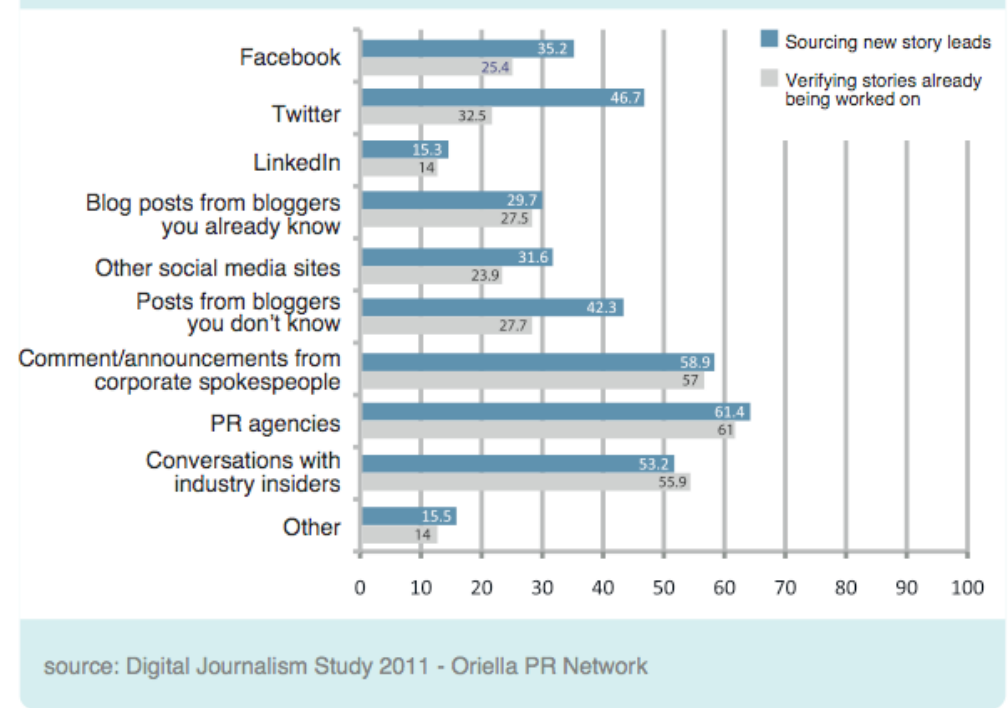

Figure 2. Pourcentage d'utilisation des réseaux sociaux comme source 
Mais bien sûr cela ne remplace pas les liens établis avec les services de presse (62\% les utilisent comme source), cela vient s'ajouter à la panoplie de vérification des faits et de repérage des faits saillants. Ces chiffres corroborent une étude pionnière conduite, toujours aux ÉtatsUnis, sur les médias sociaux à l'automne 2009 (Cision, 2009). 73\% des journalistes papier ou en ligne interrogés, déclaraient que les réseaux sociaux étaient importants ou assez importants pour enquêter et produire leurs articles. Aucun journaliste ne déclarait que les informations ainsi glanées sont plus fiables que par les canaux habituels, ils étaient même $84 \%$ à penser le contraire.

Dès lors qu'il s'agit d'appropriation d'innovations, les travaux de sociologie nous apprennent que la période initiale se caractérise par une multitude d'usages possibles, où les pratiques attendues le disputent à des choses moins anticipées. Pour essayer d'y voir clair, nous proposons ici de dresser une cartographie des usages journalistiques possibles de Twitter, grâce au croisement de plusieurs enquêtes conduites en France par notre Observatoire du webjournalisme entre septembre 2010 et mai 2012. Au début de l'année 2012, le programme de recherche Obsweb a en effet mené une large étude sur les usages des réseaux sociaux par les journalistes dont un des pans fut la réalisation d'un questionnaire en ligne à destination des journalistes français, ayant circulé au sein d'une vingtaine de rédactions par le biais des rédacteurs en chef, et qui fut mis en lien sur notre site. Avec plus de 600 réponses, ce travail permet de fournir une photographie des usages des deux principaux réseaux sociaux que sont Facebook et Twitter (Mercier \& Pignard-Cheynel, 2012). De manière attendue, les résultats font apparaître des modalités d'usage très différentes entre les deux plateformes. Ce questionnaire est complété d'une étude de comptes sociaux de journalistes durant le mois de janvier 2012 et a été précédée d'une série d'entretiens en rédaction auprès de plus d'une centaine de journalistes en 2010 et 2011.

Après avoir montré que l'utilisation de Twitter est déjà assez bien implantée dans les rédactions françaises, nous présenterons les six usages principaux que notre analyse de corpus et nos entretiens en rédaction permettent d'identifier chez les journalistes français et qui corroborent ce qui a déjà pu être observé aux États-Unis notamment. 


\section{Twitter, un outil journalistique bien plus professionnel que Facebook}

Parmi les journalistes présents sur ces réseaux sociaux, la distinction entre usage personnel et usage professionnel est fondamentale. Facebook apparaît clairement comme le réseau tourné vers l'individu et sa vie personnelle : l'usage privé représente plus de $30 \%$ des réponses à notre questionnaire en ligne, tandis qu'un peu plus de $6 \%$ des journalistes interrogés indiquent utiliser Facebook dans une perspective uniquement professionnelle. Sur Twitter, la tendance est inverse et beaucoup plus marquée : moins de 5\% des répondants déclarent utiliser Twitter dans une logique privée, tandis que 35\% en ont un usage exclusivement professionnel.

\begin{tabular}{|l|l|l|}
\hline & Facebook & Twitter \\
\hline Usage privé & $31,8 \%$ & $4,5 \%$ \\
\hline Usage professionnel & $6,5 \%$ & $35,1 \%$ \\
\hline Usage privé et professionnel & $50,1 \%$ & $49,4 \%$ \\
\hline Pas d'utilisation & $11,5 \%$ & $11,1 \%$ \\
\hline
\end{tabular}

Tableau 1. Usages privés ou publics des réseaux sociaux

On note d'ailleurs sur Facebook, une nette tendance à ouvrir davantage de comptes personnels que publics : sur la base d'un repérage de 600 comptes identifiés comme tenus par des journalistes, près de la moitié (46\%), sont privés, c'est-à-dire accessibles uniquement aux « amis ». Ce taux très élevé traduit l'usage majoritaire qui est fait de Facebook chez les journalistes : celui d'un espace d'expression personnelle davantage orienté vers la vie privée voire le divertissement et les hobbies que vers la sphère professionnelle. Avec parfois une logique de compensation professionnelle (être un peu journaliste sportif ou politique quand on est en poste dans une toute autre rubrique).

De même, la motivation à rejoindre les deux réseaux sociaux redouble cet écart entre usage personnel et professionnel. Ainsi, pour près de $60 \%$ des journalistes interrogés, c'est une utilisation personnelle préalable de Facebook qui les a conduits à envisager une utilisation professionnelle. Ce pourcentage est beaucoup plus faible (20\%) concernant Twitter, pour lequel la curiosité pour cet outil a été un puissant moteur de développement dans les rédactions. 


\begin{tabular}{|l|c|c|}
\hline & Facebook & Twitter \\
\hline Curiosité pour les nouveaux outils & $34 \%$ & $64 \%$ \\
\hline Utilisation personnelle préalable & $58 \%$ & $20 \%$ \\
\hline Incitation des confrères & $6 \%$ & $25 \%$ \\
\hline Pression de l'actualité & $7 \%$ & $16 \%$ \\
\hline Demande explicite de ma hiérarchie & $4 \%$ & $6 \%$ \\
\hline Autres & $6,5 \%$ & $3,5 \%$ \\
\hline
\end{tabular}

(Total supérieur à 100\% car plusieurs réponses étaient possibles)

Tableau 2. Les raisons ayant motivé une utilisation professionnelle de Facebook et Twitter

Lorsque l'on interroge les journalistes sur leurs pratiques de consultation et de publication sur Twitter et Facebook, on s'aperçoit que les stratégies d'usage sont différenciées voire clivantes.

\begin{tabular}{|l|c|c|}
\hline $\begin{array}{l}\text { Type de données } \\
\text { publiées }\end{array}$ & sur Twitter & sur Facebook \\
\hline Des retweets & $82,00 \%$ & \\
\hline $\begin{array}{l}\text { Des liens trouvés sur } \\
\text { le web }\end{array}$ & $75,40 \%$ & $61,60 \%$ \\
\hline Des faits d'actualité & $74,30 \%$ & $56,80 \%$ \\
\hline $\begin{array}{l}\text { Des réponses à des } \\
\text { utilisateurs de Twitter }\end{array}$ & $56,40 \%$ & $45,30 \%$ \\
\hline Ses propres articles & $53,50 \%$ & \\
\hline $\begin{array}{l}\text { Des questions - } \\
\text { interpellations à } \\
\text { des utilisateurs de } \\
\text { Twitter) }\end{array}$ & $42,30 \%$ & $32,90 \%$ \\
\hline $\begin{array}{l}\text { Des informations } \\
\text { personnelles }\end{array}$ & $15,10 \%$ & \\
\hline
\end{tabular}

Tableau 3. Types de pratiques de publication sur Twitter et Facebook

Clairement, les usages de Facebook laissent moins percevoir une dimension exclusivement professionnelle mais bien une combinatoire avec des usages plus personnels et relationnels. De façon générale, les journalistes français publient peu sur Facebook. La question étant de 
savoir si cela tient au fait que le support est encore assez neuf dans ses possibles usages journalistiques ou s'il est jugé mal adapté. Le mélange constant privé/public est un frein naturel à l'exploitation de ces profils personnels sauf à utiliser son compte Facebook personnel, comme la page officielle d'un titre de presse, en y investissant sa notoriété personnelle, comme le font Edwy Plenel et Fabrice Arfi, tous deux de Mediapart et parmi les plus actifs sur Facebook. Sur le mois de janvier 2012 étudié, seuls 30 journalistes dépassent les 100 publications sur leur compte Facebook, soit plus de 2 à 3 mises en ligne par jour, alors que de très nombreux journalistes Twitter publient plus de 10 tweets par jour.

Twitter apparaît clairement, au contraire de Facebook, comme un réseau mis au service d'une pratique professionnelle. Ainsi, ils sont $95 \%$ des répondants à déclarer utiliser Twitter pour suivre d'autres journalistes et médias, 90\% pour suivre l'actualité chaude, $85 \%$ pour faire de la veille sur des sujets et thèmes spécifiques et $84 \%$ pour rechercher des sources et des informations alternatives. Voilà pourquoi, nous considérons qu'il est plus pertinent de centrer cet article sur l'art de twitter l'actualité, si on veut saisir la façon dont les journalistes s'emparent des médias sociaux.

\begin{tabular}{|l|c|c|}
\hline & Twitter & Facebook \\
\hline Suivi d'autres journalistes et médias & $95 \%$ & $50,2 \%$ \\
\hline $\begin{array}{l}\text { Suivi de l'actualité chaude (breaking } \\
\text { news) }\end{array}$ & $90,1 \%$ & $32,3 \%$ \\
\hline Veille sur des sujets/thèmes spécifiques & $85 \%$ & $40,1 \%$ \\
\hline $\begin{array}{l}\text { Recherche de sources et informations } \\
\text { alternatives }\end{array}$ & $84,3 \%$ & $52,8 \%$ \\
\hline Veille sur votre média & $76,5 \%$ & $48,4 \%$ \\
\hline Suivi des sujets qui font le «buzz» & $65,9 \%$ & $47,9 \%$ \\
\hline
\end{tabular}

Tableau 4. Comparaison des usages de consultation déclarés de Twitter et Facebook

Ces éléments nous conduisent à conclure que l'utilité journalistique de Twitter est devenue une évidence pour beaucoup de journalistes français, sans doute parce que sa logique de fonctionnement s'y prête bien (en dépit de la limite des 140 caractères). En revanche, Facebook est d'un usage plus hybride et moins évident pour la cause journalistique. 
Il semble d'ailleurs que Facebook soit davantage investi par les titres de presse, qui tentent d'y imposer leur 'marque' sur le réseau social en fédérant une communauté la plus large possible (la page de France 24 compte plus de 760.000 fans ; celle du Monde un peu moins de 340.000) avec des utilisations très diverses de la plateforme, qui pour beaucoup s'inspirent des techniques du marketing. Twitter est lui l'outil des journalistes (plus que des médias en tant qu'institutions) qui y font preuve d'activisme et d'inventivité, les médias se contentant souvent d'utiliser Twitter comme simple vitrine en bombardant le réseau social de liens vers les articles publiés sur leur site, et souvent même de manière entièrement automatisée (et donc pas toujours pertinente).

Dressons donc désormais un panorama des usages journalistiques de Twitter, afin de montrer les potentialités que cet outil recèle pour l'information.

\section{Des usages très diversifiés de Twitter pour couvrir l'actua- lité}

À partir de notre observation systématique de 600 comptes, en janvier 2012, nous identifions six idéaux-types pour l'usage de ce réseau, sachant que les journalistes ne sont pas enfermés dans le carcan d'une seule pratique ou typologie, mais naviguent entre plusieurs usages.

\subsection{Un fort usage publicitaire ou autopromotionnel}

Dans une optique de stratégies de marque, où les contenus sont déclinés sur plusieurs supports pour élargir le lectorat potentiel, les médias sociaux sont un vecteur efficace de diffusion, que ce soit sur Facebook ou Twitter. Cela se réduit souvent à un simple placard publicitaire annonçant le titre et éventuellement un bref descriptif de ce qui est mis sur le site, sans qu'aucune dimension interactive ne soit recherchée. On observe aussi une prévalence forte de l'autopromotion sous forme de rappel de bons scores d'audience, d'annonce de rendezvous sur le support média amiral, de valorisation de ce qui est présenté comme une « exclu »... Certains organes de presse utilisent d'ailleurs un robot permettant de programmer de manière automatique l'alimentation d'un compte, en reprenant le titre de l'article suivi d'un lien URL. C'est encore beaucoup le cas dans la presse quotidienne régionale (PignardCheynel \& Sebbah, 2012). Une majorité de journalistes professionnels sur Twitter utilisent leur compte personnel en toute fidélité à leur 
organe de presse, pour faire sa publicité. Samuel Goldschmidt, grand reporter pour la radio RTL en Lorraine, a choisi comme pseudonyme @ rtlgrandest pour être clairement identifié comme membre de la radio. Il confiait en décembre 2010 aux Entretiens du webjournalisme de Metz, qu'il escomptait par ce moyen, amener de nouveaux auditeurs vers sa radio parmi ceux qui le suivent. Et dans un entretien, il assume : « J'utilise l'expression commerciale 'avantage concurrentiel' tout simplement parce que j'espère qu'à chacun de mes messages il y ait une publicité supplémentaire pour RTL. Sur Twitter, je défends la station à laquelle j'appartiens quand je vois des critiques exagérées à son sujet ».

Un nombre assez important de journalistes utilisent Twitter (ou Facebook) pour exprimer leur personnalité, leurs goûts (coup de cœur culturel ou coup de chapeau à un confrère, à un reportage) pour se valoriser (conférence publique, publication...) en espérant ainsi être repérés et imposer leur 'marque personnelle'. Il s'agit de conduire une stratégie d'existence sur les réseaux sociaux afin de gagner en notoriété, si on est précaire ou en mal d'exposition, ou de la renforcer pour d'autres. J.-C. Feraud, de Libération, pointe sa notoriété acquise grâce à Twitter et l'aide à l'évaluation d'audience qu'il en tire :

J'ai fait connaître mon blog Sur Mon Ecran Radar en grande partie grâce à Twitter : en quelques mois je suis passé de zéro à 10.000 visiteurs uniques par mois ! Sans Twitter, mon blog n'aurait pas eu ce succès puisqu'il n'est pas hébergé par le média qui m'employait jusque-là. À chaque billet, je fais un lien sur Twitter et Facebook et je vois tout de suite s'il marche au nombre de retweets ${ }^{1}$.

Un tel mouvement est connu sous le nom de personal branding que les détracteurs (nombreux dans le métier) préfèrent dévaloriser sous l'étiquette d'egobranding. En effet, un tel usage peut fortement agacer ${ }^{2}$ ceux qui plaident une éthique de l'effacement du journaliste devant les faits. Mais cela s'explique par le fait que Twitter tend à devenir un lieu de socialisation professionnelle d'un nouveau genre.

1 Entretien accordé à Raphaël Da Silva, pour son mémoire de Master 1, Informationcommunication, université de Metz, 2011.

2 M. Chahine, «Les Journalistes, rien que des brandeurs », Libération, 26 août 2010, p. 24. 


\subsection{Twitter, un nouveau 'club des journalistes'}

Afin d'avoir une idée plus fine de l'environnement dans lequel les journalistes s'insèrent via les réseaux sociaux, nous avons entrepris d'examiner leurs choix d'abonnements sur Twitter, en distinguant les plans quantitatif (nombre de comptes) et qualitatif (pertinence associée dans le cadre d'un usage professionnel). Cette cartographie des abonnements des journalistes fait apparaître des usages fortement " auto-centrés » sur la communauté des journalistes. Les personnes les plus suivies sont souvent des journalistes. Ainsi, sur presque 600 comptes de journalistes, moins de $2 \%$ ne suivent pas de confrères via Twitter et moins de 3\% jugent cela inutile. Car nos observations sur les comptes recoupent totalement le déclaratif des journalistes qui ont répondu à notre questionnaire. Ce sont aussi « les confrères d'autres rédactions » qu'ils déclarent le plus utile à suivre, loin devant d'autres acteurs sociaux. $79 \%$ jugent très utile de suivre des confrères sur Twitter contre $36 \%$ pour des hommes politiques. En croisant ces deux séries de données, on aboutit au tableau suivant :

\begin{tabular}{|l|c|c|c|c|c|c|}
\hline & $\begin{array}{c}\text { Très nom- } \\
\text { breux } \\
\text { (plusieurs } \\
\text { dizaines) }\end{array}$ & $\begin{array}{c}\text { Peu } \\
\text { nom- } \\
\text { breux }\end{array}$ & $\begin{array}{c}\text { Inexis- } \\
\text { tants }\end{array}$ & $\begin{array}{c}\text { Très } \\
\text { utile }\end{array}$ & $\begin{array}{c}\text { Peu } \\
\text { utile }\end{array}$ & Inutile \\
\hline $\begin{array}{l}\text { Confrères } \\
\text { d'autres } \\
\text { rédactions }\end{array}$ & $80 \%$ & $19 \%$ & $1 \%$ & $79 \%$ & $18 \%$ & $3 \%$ \\
\hline $\begin{array}{l}\text { Confrères de } \\
\text { votre rédaction }\end{array}$ & $38,5 \%$ & $45 \%$ & $16,5 \%$ & $36 \%$ & $46 \%$ & $8 \%$ \\
\hline Blogueurs & $40 \%$ & $52 \%$ & $8 \%$ & $53 \%$ & $38 \%$ & $9 \%$ \\
\hline $\begin{array}{l}\text { Acteurs de la } \\
\text { vie politique }\end{array}$ & $37 \%$ & $49,5 \%$ & $13,5 \%$ & $39 \%$ & $45,5 \%$ & $15,5 \%$ \\
\hline $\begin{array}{l}\text { Acteurs } \\
\text { institutionnels } \\
\text { ou locaux }\end{array}$ & $26,5 \%$ & $57 \%$ & $16,5 \%$ & $36 \%$ & $46,8 \%$ & $17,2 \%$ \\
\hline Sphère privée & $8,5 \%$ & $46 \%$ & $45,5 \%$ & $8,5 \%$ & $32,5 \%$ & $59 \%$ \\
\hline
\end{tabular}

Tableau 5. Les comptes Twitter suivis : proportion observée \& utilité déclarée 
Des résultats similaires ont été observés aux États-Unis, en 2011. «Les utilisateurs suivis le plus souvent par les editors et les reporters des dix journaux nationaux phares, sont souvent des gens comme eux : des journalistes de l'élite » (Heim, 2011, p. 18).

Les journalistes fondent au sein du réseau social Twitter une sorte de club, au sens londonien du terme. "Après Twitter, on se connaît entre happy few, journalistes et quelques lecteurs $»^{1}$. Quand on l'interrogeait fin 2010 sur les interactions sur les réseaux sociaux, un journaliste du Figaro.fr reconnaissait : «Il y a évidemment Twitter parce qu'ils nous suivent et parce qu'on les suit, et qu'on interagit, mais c'est essentiellement des confrères, des lecteurs il y en a très peu au final $»^{2}$. Malgré l'aspect ouvert des données de Twitter, ils échangent peu avec des gens qui ne sont pas de la profession ou reconnus dans la blogosphère et ne glanent que rarement de témoignages qui étoffent leurs articles grâce à Twitter. C'est singulièrement le cas pour les journalistes qui alimentent un blog réflexif sur les pratiques du métier (fréquemment enseignants dans les écoles de journalisme, comme aux États-Unis) qui font écho à leur billets mis en ligne, et trouvent sur Twitter ou leur page Facebook une communauté de pairs avec qui en débattre. Parfois ils s'interpellent entre eux, échangent par allusion, selon une logique de connivence entre ceux qui savent. Les abonnés ne sont pas exclus de ces échanges, peuvent suivre mais ils ne sont pas la cible première, voire même pas réellement nécessaires. Les comptes anglophones Twitter de Jeff Jarvis (liée à son blog) ou de Jay Rosen ou d'Erwann Gaucher et Alice Antheaume en France, sont de bons exemples. Le journaliste à Slate.fr Vincent Glad insiste sur le fait que Twitter a créé du lien social entre les webjournalistes qui ont pu ainsi se définir comme une petite communauté, partageant progressivement des règles implicites ou usages communs et s'interpelant directement sur le réseau. Twitter représente « un cadre pour rencontrer et discuter avec ses pairs, une sorte d'agora du journalisme. Avant Twitter, les journalistes web ne se connaissaient pas du tout entre eux. Maintenant, on se connaît quasi tous $»^{3}$. Twitter est une des façons de créer « un espace en commun » en profitant des capacités de «synchorisation» offertes par Internet. « La synchorisation est ce processus qui consiste à

1 Entretien Obsweb avec Antonin Sabot, Le Monde.fr, automne 2010.

2 Entretien Obsweb, avec Bastien Hugues, Le Figaro.fr, automne 2010.

3 Entretien accordé à Raphaël Da Silva, pour son mémoire de Master Informationcommunication, université de Metz, 2011. 
se donner un espace commun pour être et pour agir. La synchorisation constitue en cela le pendant spatial de la synchronisation, à savoir le processus qui consiste à se donner un temps commun pour être et pour agir » (Beaude, 2012, p. 67).

Une des façons de construire cette communauté, tout en sacrifiant à une certaine forme d'egobranding, c'est de partager sur Twitter ses humeurs, ses coups de cœur, et de faire preuve d'esprit, en étant soudés par une reconnaissance mutuelle des qualités des pairs. Cela passe aussi par la définition d'un « out-group » (selon la terminologie classique d'Henri Tajfel) : ceux qui n'en sont pas ou pas encore, ceux n'en ont pas intégré toutes les subtilités du journalisme sur Internet.

\subsection{Twitter sert aussi à se moquer de ses confrères}

La construction sociale des frontières est un élément décisif dans la définition des caractéristiques d'un groupe. Se vivre comme un club, une communauté implique de poser des lignes de démarcation qui sont naturalisées et conflictualisées dans les prises de parole publiques. Dans ce cas, les journalistes les plus actifs et les plus anciens sur Twitter (gages a priori d'une plus forte légitimité) expriment fréquemment leurs sentiments personnels sur l'actualité (coup de griffe, indignations) et cherchent à attester de leur esprit spirituel, par des saillies drolatiques sur la couverture des autres médias. Vincent Glad parle à ce titre de « journalisme LOL » ${ }^{1}$. Selon lui, la présentation des informations avec humour et légèreté se développe. Du coup, dit-il, certains des journalistes français les plus connus sur Twitter sont ceux qui font assaut de traits spirituels (drôles et/ou vachards) et pas forcément ceux qui livrent le travail d'information le plus exemplaire. Fréquemment, ce jeu consiste à chambrer des confrères, des titres concurrents ou alors des acteurs politiques et sociaux d'influence afin de prouver à tous, mais d'abord à ses pairs, que l'on ne se fait pas mener en bateau, qu'on n'est pas dupe du jeu politico-médiatique. "Quand on vient d'avoir un coup de fil « LOL », avec un homme politique, on peut en balancer un bout sur Twitter $»^{2}$.

1 Vincent Glad, «Tentative de définition du journalisme LOL », mai 2010 [en ligne], http://bienbienbien.net/2010/05/24/tentative-de-definition-du-journalisme-lol/

2 Entretien Obsweb, avec Stéphane Alliès de Mediapart.fr, automne 2010. 
Début août 2012, dans un retweet, J.C. Feraud, de Libération, tourne sévèrement en dérision l'un des titres racoleurs du magazine Elle, façon polissonneries de l'été.

RT@KlaireFr: Après "la pipe, ciment du couple", on aura peut-être

"la branlette, mortier du célibat" \#Elle \#VaSavoir

Dérouler

Figure 3. Capture écran d'un tweet de journaliste.

Durant les soirées électorales de la présidentielle française, le chambrage fut aussi une activité intense sur Twitter.

\section{narvic@narvic}

Miracle! La porte vient de s'ouvrir en direct à la TV \#correze Scoop: il

n'y a rien à voir !

Dérouler

Figure 4. Capture écran d'un tweet de journaliste.

Et les journalistes sur Internet se gargarisent de pouvoir déjà commenter des données de vote que les médias traditionnels doivent tenir sous embargo jusqu'à $20 \mathrm{~h}$.

\section{Margaux Bergey @MargauxBergey}

Pensées pour les confrères de télé qui doivent encore meubler

l'antenne pendant $45 \mathrm{~min}$ alors qu'on connaît les résultats

\#Presidentielle

Dérouler

Figure 5. Capture écran d'un tweet de journaliste.

Pendant que d'autres dénoncent, du coup, l'hypocrisie des médias traditionnels pour suggérer les résultats qu'ils ne peuvent donner.

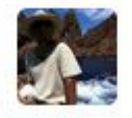

\section{CDestracque@CDestracque}

Ca me rappelle ma soirée municipale 2008 à la mairie de \#Metz

cachant une "surprise" à $19 \mathrm{H} 30$ avec des hurlements de joie derrière

\#ridicule

Dérouler

Figure 6. Capture écran d'un tweet de journaliste. 
Sur Twitter, la dérision est donc classiquement utilisée comme un instrument de contrôle social, afin de dénoncer certaines pratiques médiatiques qui ne sont pas conformes à l'éthique du métier, ou à la haute opinion que ces journalistes sur Twitter s'en font. «La dérision propose à son auditoire une nouvelle communauté, celle des rieurs. Le rire permet une identification positive à la communauté de ceux qui ont le sens de l'humour. Le rire est symbole d'individuation par excellence et l'occasion d'afficher sa liberté et sa capacité à comprendre où il fallait rire et entrer ainsi en connivence avec autrui » (Mercier, 2001, p. 12-13). Mais ce contrôle peut être plus combatif. Certains journalistes utilisent la liberté de ton critique liée au réseau pour jouer les redresseurs de déontologie. « Nous la vérification Internet, c'est notre boulot. En un quart d'heure on voit que ce n'est pas crédible. Alors que déjà 50 mecs dont des étudiants en journalisme, des journalistes d'info, un mec de France info qui le retweetent... C'est un peu facile. Personnellement je les allume sur Twitter $»^{1}$.

\subsection{La tribune libre : Twitter comme espace d'expression moins contraint mais pas sans régulations collectives}

Twitter serait aujourd'hui, ce qu'était le blog hier : un espace où les journalistes s'autorisent une liberté de ton, de choix des sujets, de jugements que leur structure de publication professionnelle ne leur donne pas et qui les valorise à leurs yeux. Un espace mi-personnel, mirédactionnel qui autorise un regard décalé. Les journalistes sur Twitter cherchent à se démarquer de leur média au profit d'une approche plus individuelle et parfois militante. Ils sont d'ailleurs nombreux à préciser dans leur notice biographique, de manière tantôt sincère tantôt ironique, que « leurs tweets n'engagent qu'eux ». L'espace d'expression ainsi créé permet le mélange des genres : production d'informations ou simples rediffusions de ce que sa veille a permis de dénicher, coups de cœur ou coup de gueule, commentaires acerbes et engagés, conseils et bons ou mauvais points distribués aux acteurs sociaux observés. Cette pratique se révèle par l'usage intensif du second degré, de l'ironie voire d'un certain cynisme. Les journalistes américains profitent des mêmes libertés du support. Dans une étude systématique sur un échantillon de tweets de mars 2010, il apparaît que «presque $16 \%$ des tweets des

1 Entretien Obsweb, avec Antonin Sabot, du Monde.fr, automne 2010. 
journalistes transmettent d'abord des opinions, s'y ajoutent $27 \%$ de tweets qui véhiculent en priorité une information mais en contenant au moins un élément d'opinion » (Lasorsa et al., 2012, p. 29).

Le journaliste sur Twitter se caractérise alors par une certaine schizophrénie : professionnel de l'information, il se doit de respecter des règles éthiques et déontologiques de rigueur, d'objectivité, de vérification des informations, il condamne certains collègues déviants sur les médias traditionnels ; twittos passionné, il peut se laisser aller à la subjectivité, aux commentaires personnels ou à une activité de retweet qui ne laisse pas le temps nécessaire au recoupement des informations.

Espace de liberté et d'affranchissement, Twitter donne donc lieu à des dérapages, et parfois à des sanctions comme ce fut le cas le 9 mai 2012 pour le commentateur sportif de RTL Pierre Salviac et sa blague sexiste à gros sabots, à propos de Valérie Trierweiler ${ }^{1}$. « Á toutes mes consœurs, baisez utile, vous avez une chance de vous retrouver première Dame de France ». Ce tweet a été intensément commenté sur les réseaux sociaux, suscitant des centaines de réactions indignées, de particuliers et de journalistes, manifestant ainsi le pouvoir d'autorégulation de cet « espace en commun ». Il est intéressant de noter à ce titre que le patron de l'information de RTL, Jacques Esnoux, s'est senti obligé de dénoncer lui aussi sur Twitter le propos injurieux de son collaborateur, avant de l'évincer, en l'interpelant directement : "Ton tweet est absolument intolérable. J'y vois un sexisme vulgaire inqualifiable que je condamne ». D'où la mise en place des premières formes de régulation.

Pour d'autres médias, il convient d'interdire l'usage jugé parasitaire de ces modes d'expression nouveaux. Canalt, considère ainsi que la non exclusivité de certains tweets ou retweets de ses journalistes s'interprète comme un défaut de loyauté, d'où l'interdiction d'utiliser Twitter. Dans un entretien réalisé à l'automne 2010, Philippe Brochen de Libération. fr, ne pouvait cacher son énervement face à un usage fautif pour la rédaction du compte Twitter personnel d'un collègue.

La semaine dernière, je découvre une info sur un autre site, une info exclusive, d'un collègue qui a rencontré un politique lors d'un déj., qui lui a dit une super info ; ce con de collègue, qu'est-ce qu'il fait, plutôt que de nous passer un coup de fil,

$1 C f$. notre analyse : «Viré pour un tweet : ce que dit le « cas Salviac» des journalistes sur Twitter », [en ligne], http://obsweb.net/2012/05/10/vire-pour-un-tweet-ce-quedit-le-cas-salviac-des-journalistes-sur-twitter/. 
pour nous donner l'info parce que lui ne va pas l'utiliser avant le lendemain, d'ici là, peut-être qu'elle sera morte, ce collègue, il le twitte, sur son Twitter; donc du coup, tous ses followers dans le métier récupèrent l'info, et on la retrouve sur un autre site ! Et elle n'est pas sur le nôtre!

Johan Hufnage ${ }^{1} n^{\prime}$ 'est ni pour la censure ni pour imposer une charte à ses journalistes qu'il juge inutile. Il compte sur l'esprit de responsabilité de chaque reporter, qui doit suffire à garantir un comportement fidèle à la maison mère. Surtout, il soutient le droit à l'erreur et à expérimenter. D'autres médias, comme l'AFP, se sont dotés au printemps 2011 d'une charte interne du bon usage ${ }^{2}$.

\subsection{Un usage informationnel et participatif : du fil info au crowd- sourcing}

Dans les usages professionnels Twitter est assimiléà un fil d'actualité prisé, pour de nombreuses raisons : la gratuité de l'accès, l'abondance des informations, l'immédiateté de la diffusion, l'identification aisée des auteurs (même si des pseudonymes sont utilisés, la page profil est une carte de visite indispensable pour que les journalistes vous retweetent et vous suivent ou pas). Certains journalistes n'hésitent donc pas à assimiler Twitter au fil d'agence AFP. Pour Samuel Goldschmidt, grand reporter à $R T L$, « ce réseau m'apporte des alertes beaucoup plus fréquentes qui font que je lis de moins en moins la presse classique. Il faut le reconnaître, sur Twitter on a tout, tout de suite et plus vite. C'est une espèce d'AFP dans la poche, y compris au niveau local ». Pour Jean-Christophe Feraud, journaliste à Libération :

La première fonction de Twitter c'est la veille. Je commence tous les matins par une revue de Twitter parallèle à ma revue de presse: que s'est-il passé cette nuit dans le monde, dans le pays, sur le secteur qui m'intéresse? Qu'est ce qui est important, qui fait le buzz, qu'est-ce qui l'est moins ? J'ai la réponse très

1 J. Hufnagel, «Pourquoi Slate.fr n'a pas de charte pour Twitter et les réseaux sociaux », juillet 2011 [en ligne], http://www.slate.fr/story/41413/slate-chartetwitter-reseaux-sociaux

$2 C f$. Alice Antheaume, « Réseaux sociaux et journalistes: l'école des chartes », juillet 2011, [en ligne], http://blog.slate.fr/labo-journalisme-sciences-po/2011/07/20/ reseaux-sociaux-et-journalistes-ecole-des-chartes/ 
rapidement. Je peux contacter en direct des sources que je ne pourrai pas toucher, ou difficilement, en d'autres circonstances. Depuis que je me sers activement de Twitter je ne regarde plus ou presque mon fil de dépêches $A F P$ ! Twitter est un assistant formidable pour ne rien rater. C'est devenu indispensable car avec l'Internet temps réel l'info devient temps réel (entretien Obsweb, automne 2010).

Vincent Glad parle aussi d'un « fil $A F P$ humanisé, à la fois instructif et addictif ». Cette addiction très évoquée dans nos entretiens est dénoncée par le journaliste de Télérama, Nicolas Delesalle, qui a décidé à l'été 2012 de fermer son compte, en s'en déclarant fort soulagé1.

De nombreux journalistes sur Twitter considèrent qu'ils ont aussi un rôle de « curateur $»^{2}$ des informations publiées sur d'autres médias, qui ne sont pas vécus comme des concurrents. Jean-Marc Manach, de Owni.fr, décrit cela comme « un journalisme de liens » qui « partage sur ces réseaux » (entretien Obsweb, automne 2010). Et il poursuit :

Il y a plein de choses que je vois, plein d'articles qui me semblent intéressants, et donc plutôt que d'en faire moi un papier, ce que font généralement la presse traditionnelle. Moi, si Libé fait un très bon papier, je vais renvoyer mes lecteurs sur les réseaux sociaux vers ce papier de Libé. Donc je partage beaucoup plus d'informations que je n'en écris. Et le boulot de journaliste sur Internet, c'est aussi ça. Je passe l'info.

Ce rôle de passeur est évidemment bien plus aisé à assumer dès lors qu'on appartient, comme ici, à une rédaction de médias NEL (nés en ligne) pour lesquels le modèle économique ne repose pas sur l'achat d'une information parue ailleurs et qui considèrent comme un service à offrir, des sélections d'articles trouvées sur la Toile.

Les médias d'information peuvent aussi facilement utiliser les réseaux sociaux pour questionner leurs abonnés. Même si c'est très loin de se généraliser, il n'est plus rare de voir des journalistes interpeler

1 N. Delesalle, Télérama, 20 juillet, 2012. doi : http://www.telerama.fr/medias/j-aidit-adieu-a-mes-6-500-followers-sur-twitter-par-koliadelesalle,84456.php

2 La curation désigne les activités d'agrégation, de tri et de classement de données. Dans l'univers du journalisme en ligne, « un "Content Curator" est quelqu'un qui continuellement cherche, regroupe, organise et partage le meilleur contenu en ligne et le plus pertinent sur un sujet» (nous traduisons). Rohit Bhargava, « Manifesto for the Content Curator », Socialmediatoday.com, 9 octobre 2009. 
sur Facebook ou Twitter une recherche de témoignage, eux-mêmes ou en passant par des sites qui ont vocation à mettre en contact des journalistes avec des sources potentielles, comme ici :

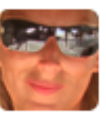

VoxForte $\odot$ VoxForte

Un journaliste (NRJ12 - Tellement Vrai ) recherche : Familles

21 Juin

homoparentales bit.ly/NjCPM8

Figure 7. Capture écran d'un tweet de journaliste.

Les médias locaux sont aussi très prompts à solliciter leurs abonnés pour en faire des sortes de correspondants locaux ponctuels (« envoyeznous vos photos de l'accident... ou des dégâts des orages d'hier », « signalez sur notre compte Facebook les bouchons routiers que vous rencontrez », etc.). C'est la version électronique, mobile et instantanée du vieux « fil rouge » d'Europel, où les témoins d'un événement étaient incités à téléphoner à la rédaction.

La production des internautes sur les réseaux sociaux peut aussi être reprise, car ils deviennent eux-mêmes sources et diffuseurs d'informations. Le premier cas marquant, aux Etats-Unis, fut le crash d'un avion dans l'Hudson, le 15 janvier 2009. Le pilote qui survolait New-York a eu le réflexe de sauver l'avion et ses passagers en amerrissant en douceur sur le fleuve. Le premier message d'information concernant cet incident a été posté sur Twitter, par un témoin oculaire, Janis Krums, qui a observé la scène d'un bateau et en a diffusé une photo placée sur son compte Twitpic.com, qui fit le tour du monde. 34 minutes après sa diffusion, il était interviewé en direct par la chaîne $M S N B C$.

\section{jkrums} Follow

\section{http://twitpic.com/135xa - There's a plane in the Hudson. I'm on the ferry going to pick up the people. Crazy.}

Figure 8. Capture écran d'un des premiers tweets de citoyen abondamment repris par les médias. 


\subsection{Un usage informationnel spécifique : la couverture-live}

Twitter va aujourd'hui plus vite que n'importe quel autre média car ses contraintes éditoriales et techniques sont très légères. Les journalistes n'hésitent donc plus à publier une première info sur Twitter, le plus en direct possible, avant de la traiter de manière approfondie pour leur média. Twitter est parfaitement adapté pour la diffusion virale des « breaking news », comme le soulignait dès 2008 un article du Read Write Web ${ }^{1}$, ou Paul Bradshaw ${ }^{2}$, confirmé par Paul Farhi ${ }^{3}$ Même si tout ceci n'est pas sans risques par rapport à l'idéal de recoupement et de vérification.

Les réseaux sociaux sont donc devenus des lieux où la couverture d'un événement peut prendre forme au point de devenir une référence pour les autres médias : les révolutions arabes en Tunisie et Egypte ou l'affaire DSK en 2011, et déjà les manifestations de 2009 en Iran qu'on a surnommées « révolution Twitter ». Twitter y a été utilisé par les journalistes comme un média, favorisant une couverture en temps réel à coup de retweets de ce que des citoyens diffusaient ou de tweets que des journalistes seuls témoins possibles d'un fait, émettaient. Souvenonsnous des journalistes de BFM TV lisant sur leur écran d'ordinateur les tweets de journalistes d'autres médias qui twittaient depuis le tribunal de New York au moment de l'audition de DSK, alors que les caméras en direct étaient interdites dans le prétoire. Cette couverture en direct via Twitter fut également de mise lors du siège de l'appartement de Mohammed Merah à Toulouse en mars 2012, comme en témoigne ce tweet (avec toujours ce ton humoristique et ce clin d'oeil à la couverture d'Astérix et Cléopâtre) de Soren Seelow, journaliste du Monde.fr:

1 M. Kirkpatrick, « How We Use Twitter for Journalism », Read Write Web, avril 2008, [en ligne], http://www.readwriteweb.com/archives/twitter_for_journalists. php

2 Bradshaw P., 2008, " How to master Twitter, if you're a journalist », Journalism. co.uk, avril [en ligne] http://www.journalism.co.uk/skills/how-to-master-twitter-ifyou-re-a-journalist/s7/a531439/

3 « News organizations and reporters have been quick to adopt Twitter for an obvious reason: Its speed and brevity make it ideal for pushing out scoops and breaking news to Twitter-savvy readers», (Farhi, 2009). 
Soren Seelow Osoren_seelow

\#Toulouse 31 h de live c'est : 230 tweets, 10000 followers, 14 recharges d'iPhone, 12 cafés, 6 bières, 3 paquets de clopes et $1 \mathrm{~h}$ de sommeil

Figure 9. Capture écran d'un tweet de journaliste.

De par l'extrême concision de ses contenus, Twitter se prête particulièrement bien aux citations et verbatim. Une ou deux phrases sont extraites d'un discours, d'une émission de télévision, etc. avec l'assurance, via Twitter qu'elles connaîtront une diffusion large et rapide. Et si les journalistes ne les repèrent pas eux-mêmes ils peuvent retweeter d'un autre compte (confrère, adversaire politique, plus rarement simple citoyen). Ce verbatim peut s'accompagner d'une remarque contextuelle ou critique sur les propos cités. Voici deux exemples tirés de la dernière présidentielle française, au printemps 2012 : « «Dix ans qu'une droite s'est installée au pouvoir et qu'elle a défait ce que nous avions construit» applaudissements nourris pour @ fhollande » (France info) ou « «Je n'ai pas voté le traité de Lisbonne»: @Bayrou réécrit l'histoire http://bit.ly/ xnStkh \#europe » (Rue89)

Cet usage a déjà trouvé son public. Nous avons observé parmi les tweets les mieux rediffusés de notre corpus de janvier 2012, beaucoup de verbatim politiques issus d'émissions ou de meetings, notamment ceux accompagnés d'un petit commentaire mordant ou sarcastique, mêlant souvent observation sur l'homme politique et sur le dispositif médiatique dans lequel il s'insère. A propos des soirées télé commentées, voici une sélection des messages issus de notre corpus qui ont dépassé la barre des 10 retweets lors d'une intervention télévisée du Président Sarkozy le 29 janvier 2012.

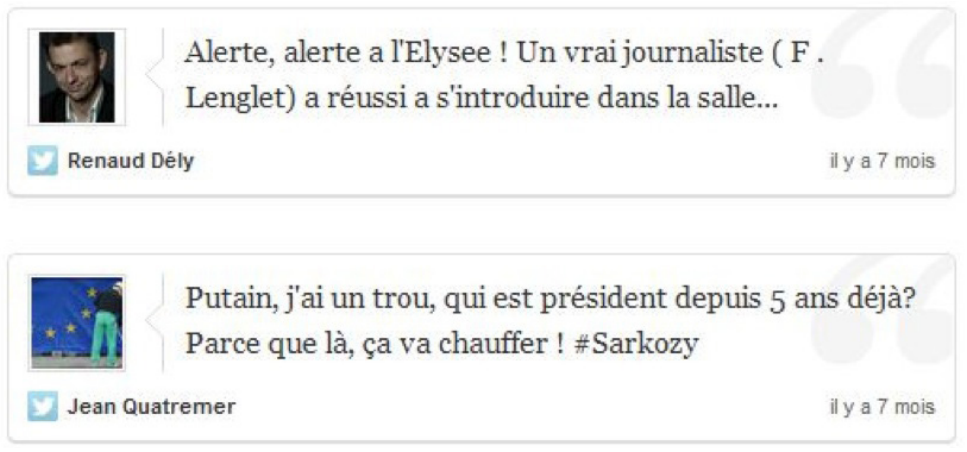

Figure 10. Captures écran de tweets de journalistes. 
Un tel succès dans l'usage est sans doute lié au fait que Twitter, pour certains hommes politiques (Cécile Duflot, Nadine Morano...) comme pour les journalistes, est très adapté (tout dire en 140 caractères) à la logique bien connue de la « petite phrase », de la formule lapidaire assassine, provocatrice ou symbolique, qui garantit un bon écho médiatique, une reprise, du «buzz».

\section{Conclusion}

Un des effets les plus notables du recours à Twitter pour les journalistes français est sans conteste un effet de réseautage. On voit dans la façon dont les journalistes en ligne s'interpellent sur ce réseau, échangent selon une logique du clin d'œil ou au contraire du coup de pied de l'âne, qu'il contribue à créer un cercle, une communauté, avec ses valeurs, ses figures emblématiques, ses pionniers, ses repoussoirs, ses codes internes. Une autre caractéristique qui se dégage, tient à la façon dont Twitter est approprié comme un espace de libération de certaines contraintes du métier. C'est un lieu où de nombreux journalistes pratiquent et revendiquent une liberté de ton, une possibilité d'éditorialiser leurs prise de parole, sur le mode du commentaire, de la critique ironique ou cinglante, à l'égard des acteurs sociaux ou des confrères. On observe aussi que la pratique participative n'est pas prioritaire dans les usages journalistiques, et que Twitter est largement autocentré et utilisé pour s'affranchir de quelques servitudes du métier, comme purent l'être les blogs d'information, à l'origine. Ces blogs, dont Jane Singer (2005) a très bien montré qu'ils furent rapidement «normalisés » par les institutions de presse, intégrés au sein des sites rédactionnels.

Le même sort attend-il Twitter ? La régulation est en tout cas en cours, de façon encore tâtonnante, avec son lot d'erreurs professionnelles, de sanctions, de mise en place de règles d'usage, voire de recours aux tribunaux. Et il faut admettre que les enjeux identitaires et déontologiques liés au traitement de l'information sur les réseaux sociaux sont réels et pas anodins. En témoignent, les tensions qui se font jour autour du journalisme sur Twitter, bien mises en évidence par Nicola Bruno (Bruno, 2011), entre volonté d'autonomisation rédactionnelle et contrôle hiérarchique et organisationnelle ; entre discours de valorisation du renouveau journalistique grâce à Twitter et dénonciation des dérapages et dérives (trop grandes expressivité, subjectivité, réactivité, risques accrus de manipulation...) à des fins de 
(re)normalisation professionnelle. Autant de pistes que nous entendons explorer en prolongement de nos actuels travaux.

\section{Références}

Ahmad, A. N. (2010). Is Twitter a Useful Tool for Journalists? Journal of Media Practice, 11(2), 145-155.

Beaude, B. (2012). Internet : changer l'espace, changer la société. Limoges : FYP éditions.

Bruno, N. (2011). « Tweet First, Verify Later? How Real-Time Information is Changing the Coverage of Worldwide Crisis Events », Reuters Institute Fellowship Paper, University of Oxford.

Cision, \& George Washington University (2009). Social Media \& Online Usage Study.

Farhi, P. (2009). The Twitter Explosion. American Journalism Review, avril/mai, [en ligne].

Heim, K. (2011). Follow the Leaders: Newspaper Journalists' Networks of Association on Twitter. Association for Education in Journalism and Mass Communication, Saint Louis, août, [en ligne].

Hermida, A. (2010). Twittering the news: The Emergence of Ambient Journalism. Journalism Practice, 4(3), 297-308.

Lasorsa, D., Lewis, S., \& Holton, A. (2012). Normalizing Twitter: Journalism Practice in an Emerging Communication Space. Journalism Studies, 13(1), 19-36.

Mercier, A., \& Dollander, T. (2012). Le Journalisme au risque de la vitesse. Les Cahiers de l'éducation permanente, mars, 9-14.

Mercier, A., \& Pignard-Cheynel, N. (2012). Les usages des réseaux sociaux par les journalistes français. Obsweb.net. doi : http://obsweb.net/2012/05/14/enquete-surles-usages-des-reseaux-sociaux-par-les-journalistes-francais/

Mercier, A., (2001). Pouvoirs de la dérision, dérision des pouvoirs. Hermès, 29, 9-21.

Pignard-Cheynel, N., \& Sebbah, B. (2012), « La presse quotidienne régionale sur les réseaux sociaux. Étude de la présence des titres français sur Facebook et Twitter ». Sciences de la Société.

Scherrer, E. (2011). A-t-on encore besoin des journalistes ? Paris : PUF. 